\title{
Word Recognition with a Hierarchical Neural Network
}

\author{
Xavier Domont ${ }^{1,2}$, Martin Heckmann ${ }^{1}$, Heiko Wersing ${ }^{1}$, \\ Frank Joublin $^{1}$, Stefan Menzel ${ }^{1}$, Bernhard Sendhoff ${ }^{1}$, Christian Goerick ${ }^{1}$ \\ ${ }^{1}$ Honda Research Institute Europe, 63073 Offenbach/Main, Germany \\ \{firstname.lastname $\}$ ehonda-ri.de \\ ${ }^{2}$ Technische Universität Darmstadt, 64283 Darmstadt, Germany \\ xavier.domontertr.tu-darmstadt.de
}

\begin{abstract}
In this paper we propose a feedforward neural network for syllable recognition. The core of the recognition system is based on a hierarchical architecture initially developed for visual object recognition. We show that, given the similarities between the primary auditory and visual cortexes, such a system can successfully be used for speech recognition. Syllables are used as basic units for the recognition. Their spectrograms, computed using a Gammatone filterbank, are interpreted as images and subsequently feed into the neural network after a preprocessing step that enhances the formant frequencies and normalizes the length of the syllables. The performance of our system has been analyzed on the recognition of 25 different monosyllabic words. The parameters of the architecture have been optimized using an evolutionary strategy. Compared to the Sphinx- 4 speech recognition system, our system achieves better robustness and generalization capabilities in noisy conditions.
\end{abstract}

\section{Introduction}

The aim of the proposed speech recognition architecture is to overcome the limitations of conventional, HMM-based, systems which substantially lack robustness against noise.

It has recently been shown that the time-frequency receptive fields in the primary auditory cortex of ferrets have strong similarities to those of the visual cortex [1]. They are selective to modulations in the time-frequency domain and have Gaborlike shapes. A mathematical model of these receptive fields was given in [2] and has already been used for source separation [3] and speech detection [4]. As Gabor-like filters are extensively used in object recognition systems [5, 6], we decided to develop a system for speech recognition by adapting the feedforward neural network initially developed by Wersing and Körner for object recognition [6].

Syllables are the basic units for speech production and show less co-articulatory effects across their boundaries. Therefore, we believe that they are the adequate speech units for our biologically-inspired system. Moreover, the syllable segmentation required for the training of the system seems biologically plausible for speech acquisition.

The building blocks of the system (Fig. 1) are detailed in the following sections. After explaining how we optimized the parameters of the architecture using an evolutionary strategy, we will compare our results to a state of the art speech recognition system and conclude with a discussion of the obtained results.

\section{Preprocessing of the spectrogram}

The preprocessing mainly aims at transforming a previously segmented speech signal, corresponding to one syllable, into an "image" that is fed into the hierarchical recognition architecture. A two-dimensional representation of a signal is obtained by computing its spectrogram. In addition to the phonetic information, the speech signal also contains many speaker and recording specific information. As the phonetic information is chiefly conveyed by the formant trajectories, we enhance them in the spectrograms prior to recognition.

We used a Gammatone filterbank to compute the spectrogram of the signal. It models the response of the basilar membrane in the human inner ear and is, therefore, adapted to a biology-inspired system. The signal's sampling frequency is $16 \mathrm{kHz}$. The filterbank has 128 channels ranging from $80 \mathrm{~Hz}$ to $8 \mathrm{kHz}$. The left part of Fig. 2 shows the response of the Gammatone filterbank after rectification and low-pass filtering. To compensate for the influence of the speech excitation signal, the high frequencies are emphasized by $+6 \mathrm{~dB}$ per octave resulting in a flattened spectrogram (Fig. 2 center). Next, the formant frequencies are enhanced by filtering along the channel axis using mexican-hat filters (Fig. 2 right), only the positive values are kept. For the filtering the size of the kernel is channeldependent, varying from $90 \mathrm{~Hz}$ for low frequencies to $120 \mathrm{~Hz}$ for high frequencies. This takes the logarithmic arrangement of the center frequencies in the Gammatone filterbank into account.

Finally, the length of the spectrogram is scaled using linear interpolation so that all the spectrograms feeding the recognition hierarchy have the same size. The sampling rate is then reduced to $100 \mathrm{~Hz}$. By doing so syllables of different lengths are scaled to the same length. This relies on the assumption that a linear scaling can handle variations in the length of the same syllable uttered at different speaking rates. However, these are known to be non-linear. In particular, some parts of the signal, like vowels, are more affected by variation in the speech rate than other parts, e.g. plosives. The generalization over these variations is a main challenge in the recognition task. In order to also assess the performance of the recognition hierarchy independent of this non-linear scaling, we applied the Dynamic Time Warping (DTW) method to the spectrograms. For each syllable, we selected one single repetition as reference template and aligned the other by DTW.

Afterwards the syllables were again scaled to the same length and downsampled. At the output of the preprocessing stage the spectrograms feeding the recognition hierarchy have 

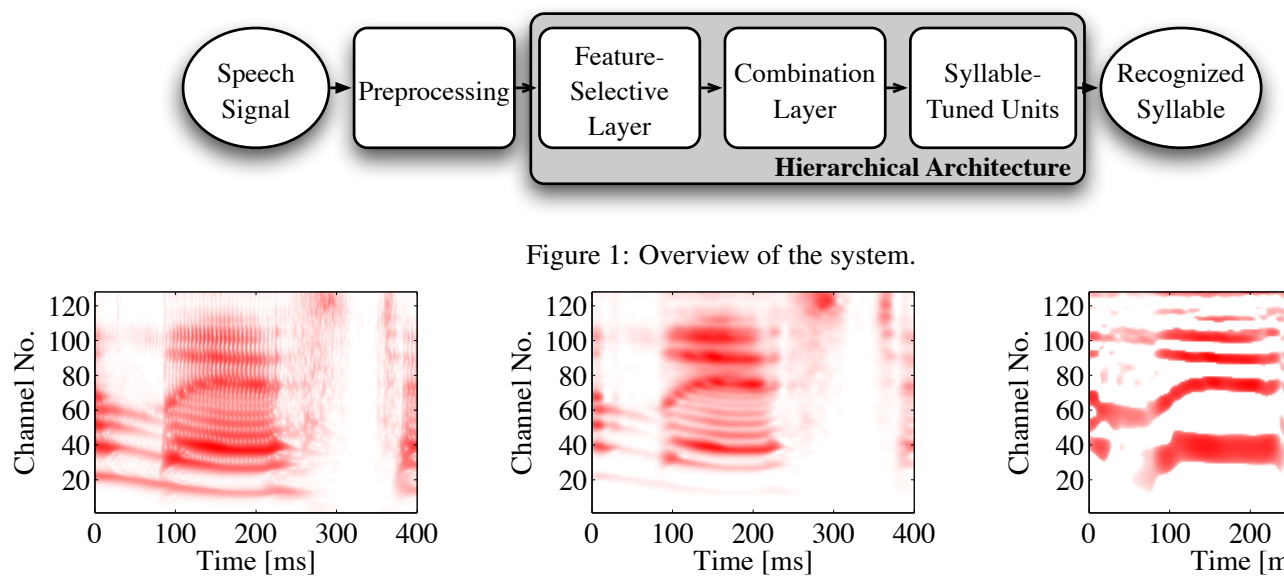

Figure 1: Overview of the system.
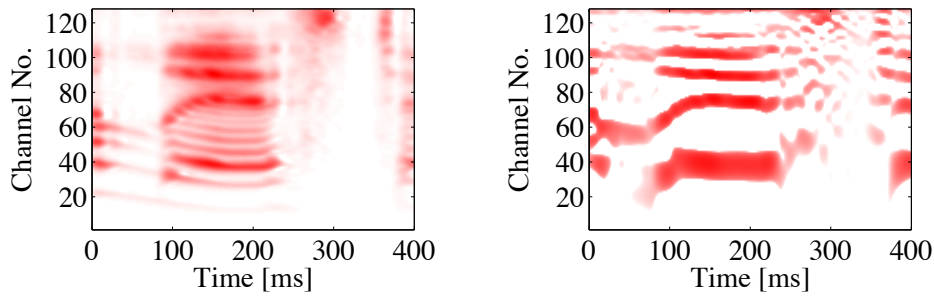

Figure 2: Overview of the preprocessing step for the word "list" spoken by a female American speaker. The 128 channels logarithmically span the frequency range from $80 \mathrm{~Hz}$ to $8 \mathrm{kHz}$. Left: Response of the basilar membrane. Center: After a low-pass filtering over time and a preemphasis has been applied. Right: The harmonic structure has been removed using a filtering along the frequency axis.

all the size of $128 \times 128$, i.e. 128 time frames over 128 frequency channels. Note, however, that the application of DTW requires that a hypothesis for the syllable is available. Thus, it cannot easily be applied to a real recognition test.

\section{The recognition hierarchy}

The preprocessed two-dimensional spectrogram is from now on considered to be an image and feeds into a feedforward architecture initially aimed at visual object recognition. However, the structure of spectrograms differs from the structure of images taken from objects and, while keeping the overall layout of the network described in [6], the receptive fields and the parameters of the neurons were retrained for the task of syllable recognition. The recognition hierarchy is illustrated in Fig. 3.

\subsection{Feature-Selective Layer}

The first feature-matching stage consists of a linear receptive field summation, a Winner-Take-Most (WTM) and a pooling mechanism. The preprocessed spectrogram is first filtered by eight different Gabor-like filters. The purpose of these filters is to extract local features from the spectrogram. In [6] the receptive fields were chosen as four first-order even Gabor filters. For syllable recognition, 8 receptive fields were learned using independent component analysis on 3500 randomly selected local patches of preprocessed spectrograms.

The WTM competition mechanism between features at the same position introduces nonlinearity into the system. The value $r_{l}(t, f)$ of the spectrogram in the $l$ th neuron of the feature-selective layer after the WTM competition is given at the position $(t, f)$ by the following equation:

$$
r_{l}(t, f)= \begin{cases}0, & \text { if } \frac{q_{l}(t, f)}{M(t, f)}<\gamma_{1} \text { or } M(t, f)=0 \\ \frac{q_{l}(t, f)-\gamma_{1} M(t, f)}{1-\gamma_{1}}, & \text { else }\end{cases}
$$

where $q_{l}(t, f)$ is the value of the spectrogram before the WTM competition, $M(t, f)=\max _{k} q_{k}(t, f)$ the maximal value at position $(t, f)$ over the eight neurons and $0 \leq \gamma_{1} \leq 1$ is a parameter controlling the strength of the competition. A threshold $\theta_{1}$ is applied to the activity $r_{l}(t, f)$. This threshold is common for all the neurons in the layer. The pooling performs a down- sampling of the spectrogram by four in both time and frequency direction. It is done by a Gaussian receptive field with width $\sigma_{1}$. The feature-selective layer transforms the $128 \times 128$ original spectrogram to eight $32 \times 32$ spectrogram feature maps.

\subsection{Combination Layer}

The goal of the combination layer is to detect relevant local feature combinations in the first layer. Similar to the previous layer it consists of a linear receptive field summation, a Winner-TakeMost and a pooling mechanism. These combination cells are learned using the non-negative sparse coding method (NNSC) as in [6], however no invariance transformations have been implemented at this stage. Similarly to Non-Negative Matrix Factorization (NMF), the NNSC method decomposes data vectors $\mathbf{I}^{p}$ into linear combinations (with non-negative weights $s_{i}^{p}$ ) of non-negative features $\mathbf{w}_{i}$ by minimizing the following cost function:

$$
E=\sum_{p}\left\|\mathbf{I}^{p}-\sum_{i} s_{i}^{p} \mathbf{w}_{i}\right\|^{2}+\beta \sum_{p} \sum_{i}\left|s_{i}^{p}\right| .
$$

NNSC differs from NMF by the presence of a sparsity enforcing term in the cost function, controlled by the parameter $\beta$, which aims at limiting the number of non-zero coefficients required for the reconstruction. Consequently, if a feature appears often in the data, it will be learned, even if it can be obtained by a combination of two or more other features. Therefore, the NNSC is expected to learn complex and global features appearing in the data. An comprehensive description of this method can be found in [7].

For the proposed syllable recognition system 50 complex features $\mathbf{w}_{i}$ have been learned from image patches extracted from the output of the feature-selective layer. At last, a WTM competition $\left(\gamma_{2}, \theta_{2}\right)$ and pooling $\left(\sigma_{2}\right)$ are applied to the 50 neurons and their size is reduced to $16 \times 16$.

\subsection{Syllable-Tuned Units}

In the last stage of the architecture, linear discriminant classifiers are learned based on the output of the combination layer. A classical gradient descent is used for this supervised learning including an early stopping mechanism to avoid overfitting. The obtained classifiers are called Syllable-Tuned Units (STUs) in reference to the View-Tuned Units used in [5] and [6]. 


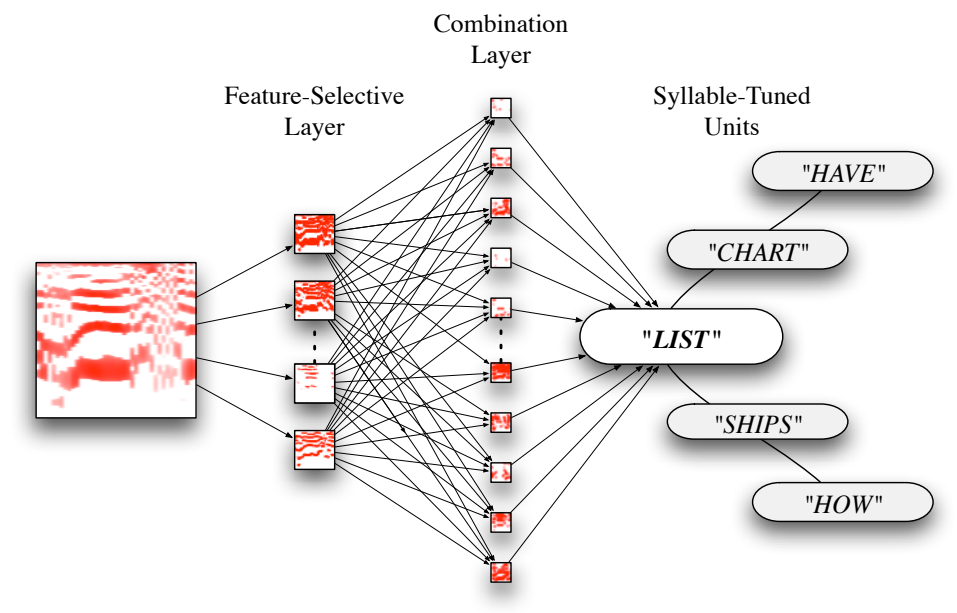

Figure 3: The system is based on a feedforward architecture with weight-sharing and a succession of feature sensitive matching and pooling stages. It comprises three stages arranged in a processing hierarchy.

\section{Optimization of the architecture}

The performance of the recognition highly depends on the choice of the non-linearities present in the hidden layers of the architecture, i.e. the coefficients and the thresholds of the WTM competitions (Eq. 1) and the width of the poolings. The six parameters $\left(\gamma_{1,2}, \theta_{1,2}\right.$ and $\left.\sigma_{1,2}\right)$ have to be tuned simultaneously and the receptive field of the combination layer as well as the Syllable-Tuned Units have to be learned at each iteration, similarly to the method used in [8].

Practically, this tuning of the model parameter set has been realized within an evolutionary optimization aiming at maximizing the recognition performance in a clean speech scenario. Due to the stochastic components and the use of a population of solutions evolutionary algorithms need more quality evaluations than other algorithms, but on the other hand they allow for a global search and are able to overcome local optima. In the present context, an evolutionary strategy with global step size adaptation (GSA-ES) has been applied relying on similar ranges of the object variables. Initially, standard values, see $[9,10]$, have been used and then tuned in some test experiments to this specific task. Based on these experiments we have chosen a population size of 32 individuals. Each generation, the two individuals with the best performance have been chosen as parents for the next generation. The optimization parameters have been scaled and the initial global step size was set to 0.003 .

Although the evolutionary optimization used a clean scenario for the performance evaluation of each individual we will show that the optimized parameters are robust with respect to noisy signals.

\section{Recognition performance}

In order to evaluate the performance of the system, a database was built using 25 very frequent monosyllabic words extracted from the DARPA Resource Management (RM) database. Isolated monosyllabic words have been chosen in lack of a syllable segmented database with sufficient size. The words were segmented using forced-alignment. For each of the monosyllabic words we selected 140 occurrences from 12 different speakers (6 males and 6 females) from the speaker dependent part of the

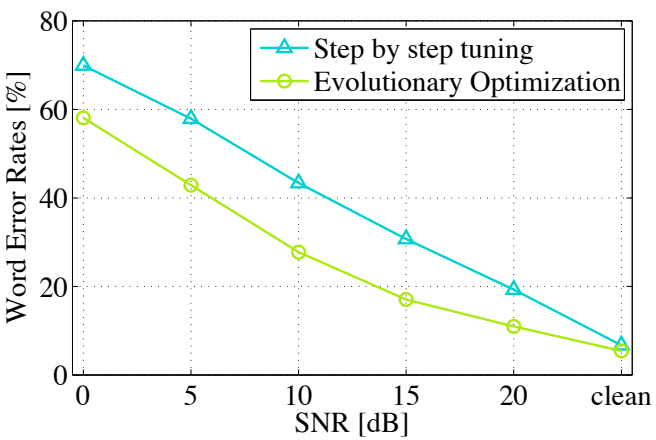

Figure 4: Improvement of the recognition performance using an evolutionary algorithm to tune the parameters, compared to manual tuning one layer after the other. The spectrograms are scaled using a linear interpolation.

database. 70 repetitions of each word were used for training, 20 for the early stopping validation of the Syllable-Tuned Units and 50 for testing.

The performance of our system has been compared to the Sphinx-4 speech recognition system, an open source speech recognition system that performs well on the whole RM corpus [11]. The Hidden Markov Models for Sphinx were trained only on the segmented monosyllabic words. The robustness towards noise has been investigated by adding babble noise to the test database at different signal to noise ratios (SNR) while training was still performed on clean data.

Figure 4 illustrates the gain in performance obtained using the evolutionary algorithm, compared to a manual tuning of the parameters one layer after the other. Following the notation introduced in [6], the optimal parameters given by the evolution strategy are $\gamma_{1}=0.82, \theta_{1}=2.66, \sigma_{1}=3.16$ for the first layer and $\gamma_{2}=0.84, \theta_{2}=2.78, \sigma_{2}=1.87$ for the second layer, when linear interpolation is used to scale the signals. Using a DTW, the optimal set of parameters is $\gamma_{1}=0.99$, $\theta_{1}=0.32, \sigma_{1}=4$ for the first layer and $\gamma_{2}=0.89, \theta_{2}=0.99$, $\sigma_{2}=1.93$. As can be seen, the performance increased due to the optimization at all SNR levels. With clean speech we ob- 

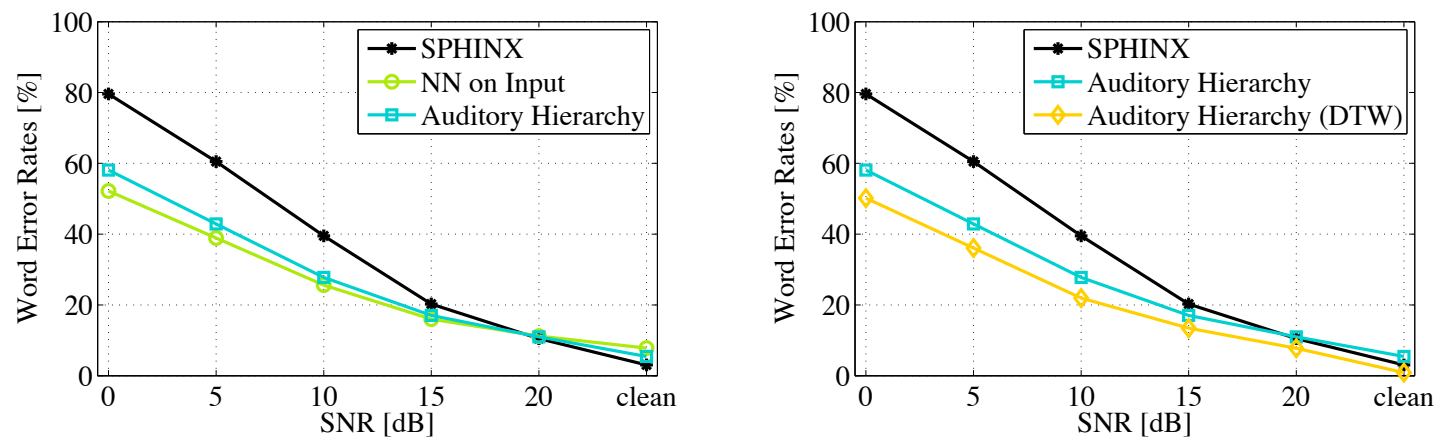

Figure 5: Comparison of the Word Error Rates (WER) between the proposed system and Sphinx-4 in the presence of babble noise. Left: The spectrograms are scaled using a linear interpolation. Comparison between Sphinx-4, a nearest neighbor classifier on the preprocessed spectrograms and the proposed hierarchy. Right: Improvement of the performance when a Dynamic Time Warping method is used to scale the signals.

serve an improvement from $6.72 \%$ to $5.44 \%$ (19\% relative). The largest improvement was achieved at $15 \mathrm{~dB}$ SNR from $30.72 \%$ to $17.04 \%$ ( $44.5 \%$ relative).

Fig. 5 summarizes the performance of both Sphinx-4 and the proposed system. To measure the baseline similarities of the image ensemble, we also give the performance of a nearest neighbor classifier $(\mathrm{NN})$ that matches the test data against all available training "views". An exhaustive storage of examples is, however, not a viable model for auditory classification. With clean signals, the STUs show better generalization capabilities and perform better than a nearest neighbor on the input layer (Fig. 5 left). For noisy signals, the STUs are slightly worse, however, at a strong reduction of representational complexity.

With a simple linear time scaling our system only outperforms Sphinx-4 in noisy conditions but shows inferior performance on clean data. When Dynamic Time Warping is used to properly scale the signals, the STUs improve the already good performance obtained directly after the preprocessing in all the cases and our system outperforms Sphinx-4 even for clean signals (Fig. 5 right). With clean data Sphinx obtains a 3.1\% Word Error Rate (WER), our system achieves $0.9 \%$ WER with the DTW and $5.4 \%$ without the DTW.

\section{Discussion}

In this paper, we presented a novel approach to speech recognition interpreting spectrograms as images and deploying a hierarchical object recognition system. To optimize the main free parameters of the system, we used an evolutionary algorithm which allows us to quickly change the system without the need for manual parameter tuning.

We could show that our system performs better than a state of the art system in noisy conditions even when we applied a simplistic linear scaling of the input for time alignment. When we aligned the current utterance with the DTW to a known representation in an optimal non-linear way, we obtained better than state of the art results for all cases tested. However, in its current form the DTW makes use of information not available in real situations.

From this we conclude that our architecture and the underlying features are more robust against noise than the commonly used mel frequency cepstral coefficients (MFCCs). This robustness against noise is very important for real world scenarios which are usually characterized by significant background noise and variations in the recording conditions. A similar robustness was also observed for visual recognition in clutter scenes [6]

Our comparison between the linear scaling and the DTW shows that the performance of the model could be significantly improved by better temporal alignment. We therefore consider methods for improving this alignment as interesting future research directions.

\section{References}

[1] S. Shamma, "On the role of space and time in auditory processing," Trends Cogn. Sci., vol. 5, no. 8, pp. 340-348, 2001.

[2] T. Chih, P. Ru, and S. Shamma, "Multiresolution spectrotemporal analysis of complex sounds," J. Acoust. Soc. Am., vol. 118, pp. 887-906, 2005.

[3] M. Elhilali and S. Shamma, "A biologically-inspired approach to the cocktail party problem," in Proc. ICASSP Conf., vol. 5, 2006, pp. V-637-640.

[4] N. Mesgarani, M. Slaney, and S. Shamma, "Discrimination of speech from non-speech based on multiscale spectro-temporal modulations," IEEE Trans. Speech and Audio Process., pp. 920-930, 2006.

[5] M. Riesenhuber and T. Poggio, "Hierachical models of object recognition in cortex," Nature Neuroscience, vol. 2, pp. 1019-1025, 1999.

[6] H. Wersing and E. Körner, "Learning optimized features for hierarchical models of invariant recognition," Neural Computation, vol. 15, no. 7, pp. 1559-1588, 2003.

[7] P. O. Hoyer, "Non-negative matrix factorization with sparseness constraints," J. Machine Learning Research, vol. 5, pp. 1457-1469, 2004.

[8] G. Schneider, H. Wersing, B. Sendhoff, and E. Körner, "Evolutionary optimization of a hierarchical object recognition model," IEEE Trans. Syst., Man and Cybern. B, Cybern., vol. 35, no. 3, pp. 426-437, 2005.

[9] H.-P. Schwefel, Evolution and Optimum Seeking. John Wiley and sons, New York, 1995.

[10] T. Bäck, Evolutionary Algorithms in Theory and Practice. Oxford University Press, 1996.

[11] W. Walker, P. Lamere, and P. Kwok, "Sphinx-4: A flexible open source framework for speech recognition," Sun Microsystems Inc., Tech. Rep., 2004. 